\title{
LA RADIO : UN MÉDIA DÉMOCRATIQUE ? UNE MISE EN PERSPECTIVE FRANÇAISE ET INTERNATIONALE
}

\author{
Jean-Jacques CHEVAL ${ }^{1}$
}

Il y a 27 ans, le Rapport MacBride, réalisé et publié à la demande de l'Unesco relevait que "dans toutes les régions du monde, la radio est le média le plus répandu. Dans les pays en développement, seule la radio peut être qualifiée de moyen de communication de masse. Une très vaste proportion de l'humanité peut aujourd'hui recevoir des émissions et possède les moyens de les capter. Aucun autre moyen de communication ne peut atteindre autant de personnes à la fois, aussi efficacement, aux fins d'information et d'enseignement, de diffusion de la culture et de récréation. La radio peut être utilisée facilement et économiquement pour atteindre des régions écartées et pour communiquer dans les nombreuses langues vernaculaires souvent sans écriture, qui existent dans les pays en développement. Presque tous les pays sont en mesure de produire des programmes radiophoniques conformes à leurs besoins politiques, à leurs modèles culturels et à leurs valeurs fondamentales.

1 Président du GRER - Groupe de Recherches et d'Études sur la Radio [http: //www. grer.fr], coordinateur du réseau IREN - International Radio Research Network, Maître de conférences, Université Michel de Montaigne - Bordeaux 3, CEMIC (centre d'Études des médias, de l'Information et de la Communication), EA 532 chevaly@club-internet.fr

Recherches en communication, $\mathrm{n}^{\circ} 26$ (2006). 
La radio est peut-être de nos jours, le moyen d'information qui a le moins subi l'emprise de la trans-nationalisation, tant pour les formes de propriété que pour la nature des programmes » ${ }^{1}$.

En 2007, ces constats conservent leur pertinence et leur réalité. On doit se rappeler que la radio reste encore à l'heure actuelle le média le plus répandu à travers le monde, celui dont l'internationalisation puis la mondialisation ont été le plus rapidement réalisées même si des inégalités demeurent toujours ${ }^{2}$. Permettant de joindre le plus de personnes à la fois, la radio est aussi l'instrument de communication moderne le plus accessible aux populations elles-mêmes. Culturellement, la radio contourne la question de l'alphabétisation, en général, et aussi, en particulier, de l'alphabétisation dans les langues dominantes. Elle peut ainsi permettre l'expression directe des langues vernaculaires, devenant de fait un instrument de résistance contre la globalisation, un instrument de défense du pluralisme culturel, avec ses valeurs démocratiques induites.

Économiquement, du fait de coûts d'investissements initiaux moindres, la multiplication de stations de radios (notamment au niveau local) est souvent envisageable quand ne s'y opposent pas des obstacles politiques, réglementaires ou économiques, et d'une autre manière, quand il s'agit de dépasser le cadre de la création des infrastructures, pour passer à celui du fonctionnement et de l'inscription dans la durée.

Là où est présente la radiodiffusion privée, le plus souvent le constat d'une moindre concentration économique des systèmes radiophoniques reste une donnée d'évidence. Si l'on se cantonne au domaine européen, le constat est qu'entre l'époque du rapport MacBride et aujourd'hui, l'augmentation et la diversification des structures radio-

1 Sean MacBride, "Voix multiples, un seul monde, communication et société aujourd'hui et demain », Paris : La Documentation Française / Nouvelles Éditions Africaines / UNESCO, 1980, p. 74-75.

2 Les taux d'équipement varient largement selon les pays ou les régions du monde, selon les sources également. Par exemple en France et 2006, selon l'institut Médiamétrie, 98,3\% des personnes âgées de 13 ans et plus possédaient au moins un appareil permettant d'écouter la radio, voiture incluse et les foyers français étaient équipés de près de 6 appareils radio en moyenne $(5,8)$. Pour l'UNESCO le nombre de postes récepteurs pour 1000 habitants n'était que de 53\%o en Haïti en 1997, 408\%o pour l'ensemble de l'Amériquue latine à la même date, mais se situait, selon la même série statistique, à un niveau de 946\% pour la France, et de $2116 \%$ aux Etats-Unis. Sources : http://www.mediametrie.fr/ et http://www.unesco.org/culture/ worldreport 
phoniques se sont imposées dans de nombreux pays. Il en va de même en Afrique sub-saharienne depuis les années 1990. Ceci ne veut pas dire que de grands groupes radiophoniques, ou que des groupes multimédias intégrant une forte dimension radiophonique, n'existent pas. Les champs traditionnels des radiodiffusions publiques ont été entamés par l'avancée des secteurs privés et commerciaux. Le tiers secteur radiophonique doit encore souvent se battre pour affirmer sa place, sa légitimité et assurer ses moyens d'existence. Néanmoins, la tentation peut être grande effectivement de faire de la radio un média démocratique par ses qualités intrinsèques. Elles sont souvent rappelées et synthétisées de la manière suivante : modicité, simplicité, rapidité, mobilité...

Dès ses origines, Bertolt Bretch avait souligné les formidables potentialités de la radio au service de l'interactivité et d'une subversion des figures, déjà classiques, de l'émetteur et du récepteur, mais c'était pour signaler dans le même temps combien ces potentiels étaient peu mis en œuvre. Ses réflexions sur la question ont été souvent invoquées par les militants des radios libres des années 70. Employée au service de projets alternatifs qui dépassaient le cadre de la radio elle-même, les stations étaient conçues comme des instruments du changement social et politique

Mais, c'est dès le début du $X^{\mathrm{e}}$ siècle, que la radio fut utilisée comme arme de subversion. À Pâques 1916, les rebelles républicains irlandais s'emparaient des équipements TSF de la Grande Poste centrale (la GPO) de Dublin où ils avaient établi leur quartier général pour avertir le monde du déclenchement de leur mouvement révolutionnaire contre l'Empire britannique. Il ne s'agissait sans doute encore que de télégraphie sans fil, mais le rôle de la radio en temps révolutionnaires était posé. C'est encore de TSF qu'il s'agit quand, en Russie, en octobre 1917, les Bolcheviques utilisent les ondes hertziennes pour relayer la révolution des Soviets de Saint Petersbourg vers Moscou, puis à travers l'immense empire russe. Et dans les décennies suivantes, et partout à travers le monde, le rôle politique de la radio ne s'est jamais démenti.

La radio a été au service des révolutions, des luttes de libération et de décolonisation. On connaît le témoignage et les réflexions de Frantz Fanon sur la station « La voix de l'Algérie libre » ou « La voix de l'Algérie combattante », durant la guerre contre la France pour l'indépendance algérienne. Selon lui, la création de cette radio a été une étape importante dans la prise de conscience des populations en les faisant 
participer plus avant au processus révolutionnaire de lutte anticoloniale ${ }^{1}$. Annoncée par avance, elle provoqua une vente massive de récepteurs, les stocks algériens étant dévalisés en moins de 20 jours. Frantz Fanon, révolutionnaire et psychothérapeute, insistait sur l'appropriation par la population algérienne d'un instrument qui lui était jusque-là étranger ; la radio était « un mauvais objet, anxiogène et maudit ». Par la suite elle a été intégrée dans un processus de formation de la conscience nationale. "La technique étrangère, "digérée » à l'occasion de la lutte nationale, est devenue un instrument de combat pour le peuple et un organe protecteur contre l'anxiété ».

L'Amérique latine offrirait pléthore d'exemples d'utilisation de la radio en temps révolutionnaire de la «Radio Rebelde » fondée, notamment par Che Guevarra, depuis les territoires libérés de la Sierra Maestra à « Radio Venceremos » au Salvador ${ }^{2}$.

En France aussi, sur le territoire national, les Français connaissent l'usage fait de la radio par la Résistance lors de la seconde guerre mondiale. Depuis Londres puis d'Afrique, alors «les Français parlent aux Français ». La figure du général de Gaulle s'adressant au pays occupé, le 18 juin 1940 appelant à la Résistance, participe depuis lors à l'histoire et à l'imaginaire national français et incarne une figure emblématique de son patrimoine. La radio est de fait assimilée à un instrument de libération, de lutte contre l'oppression. Cette figure n'est pas unique et se retrouve dans de nombreux autres pays européens, en Norvège tout particulièrement par exemple, ou bien en Pologne.

La radiodiffusion parlée à cette époque, et en fait pleinement depuis la guerre civile et la révolution espagnoles de 1936-1939, n'est plus seulement une arme d'affrontement idéologique, elle est devenue une arme de guerre, d'affrontements directs. Ce qui peut nous paraitre particulièrement intéressant fut son utilisation au service d'une légitimation politique délocalisée. L'émigré de Gaulle, qui avait quitté le territoire national, fuyard et proscrit pour avoir déserté selon les maîtres du régime de Vichy, conserva un lien quasi charnel avec le peuple français par ses adresses à la nation soumise. Face au maréchal Pétain, par la voix, il tisse un lien personnel avec les Français occupés. Comme le

1 Frantz Fanon, L'An V de la révolution algérienne, Paris : Maspéro, 1959

2 Voir par exemple Ricardo Martinez Victores, 7RR, la historia de Radio Rebelde, La Havane : Editorial de Ciencias Social, 1978 ; Carlos Henríquez Consalvi, dit «Santiago », La Terquedad del Izote, La historia de Radio Venceremos, El Salvador: Ediciones Museo de la Palabra y de la Imagen, 2003. 
Sertorius de Corneille s'exclamant : "Rome n'est plus dans Rome, elle est toute où je suis », Le général de Gaulle s'arme du verbe radiophonique et, partout où sa voix porte, il justifie son combat et sa position à travers l'utilisation d'une technique, d'un média sans qu'importe le lieu d'où il s'exprime, il incarne la France, il est la France et il affirme sa légitimité politique.

Mais le rôle de la radio au service du jeu politique ne s'inscrit pas que dans des périodes de crises traumatiques, d'affrontements. Au cours de périodes courantes de la vie démocratique on a aussi pu célébrer les vertus démocratiques de la radio.

Ainsi, à propos d'autres lieux, d'autres circonstances, les auteurs des États-Unis évoquent l'usage de la radio fait par le Président Franklin D. Roosevelt dès 1933. En s'appuyant directement sur l'opinion publique par des interventions régulières, le président a su contrer les oppositions à sa politique du New Deal. À travers ses « Fireside chats », "Roosevelt personnalisait à la fois lui-même et son auditoire et engageait directement chaque auditeur dans un rapport fait d'intimité personnelle et de communauté nationale ». Il créait une relation intime entre le Président et le peuple états-unien. Il lui promettait qu' "Ensemble, nous ne pouvons pas échouer ${ }^{1}$.

Prenant exemple sur Roosevelt, cette relation intime et personnelle, un homme politique français, Pierre Mendès France, l'institua également en 1954 au service de sa politique, durant sa Présidence du conseil. Notamment pour faire admettre au pays la fin de la première guerre d'Indochine et la défaite qu'elle représentait.

"Toutes les semaines, c'est une complicité renouvelée entre les Français et leur Président du Conseil qui leur parle [...] Mendès France dialogue d'égal à égal, parlant à chacun comme le grand frère informé et responsable soucieux du bien-être de la famille, Mendès France est donc à la fois pédagogue et capitaine d'équipe ». Partant d'une autre tradition politique, Mendès rejoint pourtant celle de Gaulle. Leurs usages de la radio témoignent de la «même personnalisation $d u$ pouvoir (c'est un homme qui gouverne et non un gouvernement), même

1 Joy Elizabeth Hayes, Assistant Professor of Communication Studies, University of Iowa « Did Herbert Hoover Broadcast the First Fireside Chat? Rethinking the Origins of Roosevelt's Radio Genius », http://www.uiowa.edu/ humiowa/jhayes. htm. On peut écouter les « Fireside Chats » de Franklin D. Roosevelt notamment à partir du site Internet suivant: http://www.mhric.org/fdr/fdr.html (D. Roosevelt Presidential Library, Hyde Park, New York). 
dialogue direct avec le peuple, même méfiance à l'égard des partis et de l'Assemblée nationale, même goût pour le geste spectaculaire destiné à frapper les imaginations et plus encore à faire revenir une part de rêve dans le politique ${ }^{1}$.

Mais si ceci participe à l'évidence d'une science du politique, s'agit-il pour autant strictement de démocratie ? Mis au crédit de la radio, ces rappels historiques ne doivent pas laisser dans l'ombre d'autres usages parallèles. Ainsi à l'époque de Roosevelt, la radio était mise à profit par le populiste et démagogue gouverneur de Louisiane Huey Pierce Long ou par son allié le prêtre Charles Edward Coughlin, autant anticommuniste qu'antisémite viscéral. De Roosevelt à Long ou Coughlin, au-delà des discours et des contenus portés par la radiodiffusion, c'était finalement la capacité de la radio à permettre un contact avec les populations en dehors des instances et des partis politiques, de tous les intermédiaires et finalement au-delà des règles jusqu'alors habituelles du jeu politique qui était en jeu. En s'invitant dans l'intimité des auditeurs, Roosevelt conviait l'ensemble des États-uniens à partager, de manière conviviale et complice, la supposée chaleur d'un feu de cheminée dans un salon de la Maison Blanche, pour mieux les convaincre du bien fondé de sa politique. Cette relation directe, ne pouvait-elle pas aussi être qualifiée de simple procédé ou de simulacre trompeur à l'aube d'une démocratie audiovisuelle où le vecteur l'emporte sur le message, les recettes marketing sur l'idéologie ? La radio a aussi contribué à cela, avant d'être très largement dépassée par la télévision en la matière.

La capacité de la radiodiffusion à transcender les barrières de toutes natures, géographiques, politiques ou sociales a également fait partie de la construction d'un imaginaire radiophonique, collectif et diffus. Elle en ferait, par excellence, un outil sinon démocratique du moins de rapprochement, de dialogue et de compréhension entre les peuples.

Dès les années 30, la puissance et la qualité des matériels le permettant, le public de la radio a pu devenir cosmopolite et écouter les programmes étrangers. La radio prend part à la création d'un mythe moderne. Certains imaginent en elle un instrument de fraternité entre les peuples. S'inscrivant dans la lignée des positivistes du XIX ${ }^{\mathrm{e}}$ siècle, les progrès techniques et humains, les inventions et leurs applications

1 La République du choix, Pierre Mendès France, 50 ans après... (2005)

http://mapage.noos.fr/republiqueduchoix/ 
sont perçues de façon utopiste et résolument optimiste. La radio semble être par nature un instrument de paix et on lui assigne le pouvoir, la faculté de gérer sinon de régler les grands problèmes de l'heure. Un texte prononcé par Sacha Guitry le 25 avril 1932, lors d'une soirée radiodiffusée justement, témoignait de ce sentiment.

«(...) Vraiment c'est effarant. De penser qu'on m'entend peut-être à Téhéran!... De penser que des gens tout à fait différents. De race et de pays, de langage et de classe, sont à l'écoute en ce moment... Le riche en son palais, l'artisan, l'ouvrier, le paysan dans sa chaumière, le poète dans son grenier... Le matelot sur son voilier... J'en imagine des milliers... Et j'en vois sur toute la terre !... (...) Et c'est, en vérité, troublant quand on y pense : être une voix qui vient de France et qui s'adresse au monde entier! Petit appareil merveilleux qui revient d'Italie et qui nous vient des cieux, réalité qui tout à coup se change en rêve, j'imagine qu'on doit te bénir à Genève... car tu fais plus pour le bonheur du genre humain que tu ne crois! (...) On n'en est pas encore à se tendre la main mais, grâce à toi, déjà comme on tend bien l'oreille »1.

Les cadrans des postes récepteurs à lampe avec leurs indications géographiques multiples induisent par eux-mêmes ses vagabondages de l'esprit. On n'écoute pas une fréquence mais on se cale sur Paris, Hilversum, Londres ou Berlin ${ }^{2}$. Puis ce sera Radio Moscou, Pékin ou Tirana pour certains, quand, dans d' autres pays, sous d'autres régimes, c'était la Voix de l'Amérique ou Radio Free Europe.

On peut par exemple ici se référer au témoignage de Eduardo Uriate Romero, militant basque, condamné à mort au procès de Burgos, en 1970, par le régime franquiste, puis grâcié : «Ma maison était un autre monde, où l'on écoutait à deux heures et quart la BBC en espagnol et à huit heures du soir la Pirenaica... à dix heures et quelques à nouveau la BBC et plus tard Radio Paris... Radio Prague... Radio Moscou... [...]» 3. Qu'importe que la station Radio España Independiente, Estación Pirenaica ou Radio Euskadi émette de Moscou, de Bucarest, de Caracas ou des frontières nord de l'Espagne, par ces nom et leurs écoutes clan-

1 Cité par René Duval, Histoire de la radio en France, Paris, Alain Moreau, 1980, p. 129.

2 Andreas Fickers, «Visibly Audible. On the symbolic representation and imagined construction of the European broadcast space in the 1930s ». Transnational Infrastructures and the rise of Contemporary Europe (TIE), First national workshop, Eindhoven University of technology, 20-23 avril 2006. (http://www.tie-project.nl/).

3 Gregorio Morán, Los españoles que dejaron de serlo, Barcelona, Ed. Planeta, 1982. 
destines, il s'agissait de conjurer les distances géographiques qui séparaient émetteurs et récepteurs, résistants antifranquistes de l'intérieur et de l'extérieur ${ }^{1}$.

Politique ou non, la littérature est pleine de références à ces pouvoirs magiques et libératoires de la radio. On peut encore ici citer l'Espagnol Antonio Muñoz Molina, dont l'œuvre romanesque, inspirée de ses propres souvenirs, évoque souvent la radio de son enfance ou de son adolescence, comme une ouverture sur le monde et moyen de s'échapper à la pesante atmosphère de l'Espagne des années 60.

« Sur le cadrans lumineux de la radio où sont inscrits les nombres et les noms des station, des villes, et des pays lointains qui émettent aussi, une main déplace lentement le sélecteur et l'aiguille survole les lieux d'une géographie inaccessible, s'arrête sur une musique qui se confond d'abord avec des coups de sifflet, des accents étrangers, un bruit sourd de papiers déchirés, la musique d'une réclame, d'une chanson ou d'un feuilleton, est-il possible qu'il y ait des gens dans une aussi petite boîte, comment arrivent-ils à se tasser [...] et on entend dans la grande pièce le frottement lent des sabots d'un cheval, un grincement de roues métalliques sur des pavés fouettés par la pluie d'un hiver éloigné venu d'un autre siècle, d'une autre ville, non seulement la radio est pleine de gens, mais il y a des chevaux et il y pleut, Paris, dit le speaker, mais je ne l'écoute plus, [...], regardant cette lumière énigmatique jaillie des profondeurs de l'appareil, un rai de lumière comme celui qu'on verrait briller sous une porte, dans une maison fermée et uniquement habitée par des voix, par toutes les voix impossibles du monde $[\ldots] »^{2}$.

Diverses, Il s'agit là sans doute d'expressions, à des degrés variables, d'une idéologie de la communication. Dans les années 30, les prophètes de la radiodiffusion inventaient avant l'heure le mythe du village global et en même temps sa lecture lénifiante. L'illusion technologique, les effets unidirectionnels que l'on prête à la radiodiffusion annoncent d'autres mirages ultérieurs. Mais ces utopies de la communication évacuaient, ou tout à la fois surévaluaient, les usages réels de la radio au service luttes politiques.

1 Marcel Plans, « Radio España independiente, la « Pirenaica », entre el mito y la propaganda », p. 114-130 in De las ondas rojas a las radios libres, textos para la historia de la radio / Lluís Bassets ed., Barcelona, Gustavo Gili, 1981.

2 Antonio Muñoz Molina, El Jinete Polaco (Le royaume des voix), Madrid, Ediciones Platneta,1991. 
Le rôle de la radio durant la seconde guerre mondiale se chargea bientôt de rappeler que comme tout autre outil technologique, comme tout autre média, la radio était fondamentalement ambivalente. Elle pourrait tout autant servir à la résistance, à la libération, qu'aux totalitarismes, à l'oppression et à la propagande mensongère et brutale, servir les préparatifs puis les usages guerriers.

Si fallait encore un rappel de ceci et si l'on peut juger totalement excessive l'assertion célèbre de Marshall McLuhan selon laquelle Hitler n'aurait pas existé sans la radio, on ne peut nier qu'elle a servi ses ambitions, ses actions et son personnage. L'efficacité des mises en scènes sonores montées par les nazis, les succès initiaux de la politique radiophonique de Joseph Goebbels, participent aussi intrinsèquement de l'histoire de ce média que ses épisodes héroïques.

"Vous n'aurez rien appris de nos illusions, rien de nos faux-pas compris, a pu écrire le poète Aragon en $1960{ }^{1}$ et, dans les années 70, les constats des périodes précédentes semblaient déjà effacés quand les militants des radios libres, seulement quelques années plus tard, réinventent une utopie radiophonique pratique.

En 1980, soulignant l'émergence de médias locaux et alternatifs le rapport MacBride pose que "L'intérêt porté aux petits médias n'est pas étranger au changement en cours dans les stratégies globales de développement. Celles-ci s'écartent, en effet, des modèles des dernières décennies en ce qu'elles mettent l'accent sur une participation plus active des collectivités dans l'expression de leur existence propre et de leurs besoins spécifiques. [...] Dans les pays industrialisés, on prend également de plus en plus conscience de l'utilité de ce type de médias, en particulier pour les minorités, les groupes ayant des intérêts spécifiques et les activités communautaires ou politiques. Les populations de ces pays se trouvent aux prises avec des problèmes d'environnement et de pollution, avec la crise de l'énergie, le chômage, l'adaptation à l'évolution technique, et bien d'autres problème du même ordre. Elles éprouvent le besoin urgent de s'exprimer avec des moyens qui soient réellement à leur disposition » ${ }^{2}$.

Radio Alice, Radio Popolare et d'autres en Italie, les stations qui suivent leur exemple en France se rattachent aux grands mouvements de contestation de l'époque. Si les radios libres militantes n'émanaient

1 Louis Aragon, «Épilogue » in Les Poètes, 1960.

2 Rapport MacBride, Op. Cit., 1980, p. 68-70. 
pas que de groupes politiques radicaux et bien identifiés, elles étaient les fruits d'éléments conjoncturels divers, de mouvances composites, voire contradictoires. Elles peuvent être analysées comme l'avatar du mouvement qualifié génériquement de Mai 1968 et, à la fois, comme le témoignage du reflux ou de l'adaptation de celui-ci, mais au final comme le résultat d'un constat d'échec. Faute d'avoir pu changer, transformer la société dans son ensemble, les militants et héritiers de 68 se tournèrent vers des expérimentations limitées mais réelles, vers des espaces disponibles et réduits, où pouvait se concevoir une alternance à l'ordre du monde ${ }^{1}$.

La critique des médias formait une partie des arguments de la contestation et celle-ci était d'autant plus vive que ces médias étaient perçus comme le miroir global d'une société récusée et comme les instruments privilégiés de l'aliénation culturelle, sociale et politique. Les services publics radiophoniques, souvent dotés de monopoles, étaient eux-mêmes perçus comme des instruments de propagande aux mains des gouvernements et des outils d'oppression au service d'une classe dominante, des « appareils idéologiques d'État », selon l'expression qu'affectionnaient les disciples du philosophe marxiste français Louis Althusser.

À l'inverse, les radios libres apparaissent comme des leviers efficaces et disponibles pour armer les militants du changement social. De fait, le dispositif idéologique qui escorte l'émergence des radios libres se nourrit d'une conception subversive des médias alternatifs qui leur serait quasiment et naturellement liée. Des théoriciens du mouvement découvrent une adéquation parfaite entre l'outil et le rôle qu'ils veulent lui voir jouer. Dans la revue française Interférences, on pouvait lire : «La radio est le meilleur médium de deux points de vue : d'une part son faible coût par rapport à sa pénétration, d'autre part sa souplesse, c'est-à-dire son accessibilité (...) n'importe qui disposant d'un magnétophone à cassette (450 francs)[69 euros] peut produire des messages sonores et dans n'importe quelle situation (exemple : usine en grève). Le seul véritable problème est politique. Aujourd'hui c'est celui du monopole qui est d'abord et avant tout le monopole des classes dirigeantes sur les moyens de communication et d'information » ${ }^{2}$. Plus largement, la communication devient le remède aux maux sociaux et politiques.

1 Armand et Michèle Mattelart, Penser les médias, Paris, Éditions La Découverte, 1986, pp. 175-180.

2 Interférences, $\mathrm{n}^{\circ} 1$, décembre 1974. 
Cette croyance dans le pouvoir libérateur des médias, en parallèle avec l'animation sociale, et par leur seule présence, pose le postulat d'une autonomie des outils socioculturels, des instruments de communication. Comme d'autres, les militants des radios libres superposent l'illusion techniciste qui "surestime la force de transformation des rapports sociaux liée à la technique » et une idéologie politique des médias qui "surestime la capacité d'imposition d'un usage social sur un stock donné d'outils techniques » ${ }^{1}$. À la décharge de ces utopistes des médias, il faut considérer que l'illusion de la capacité des radios libres à changer le monde, à bouleverser les rapports sociaux, était largement confortée et légitimée par la frayeur tout autant irraisonnée que leur seule existence provoquait en retour chez les tenants de l'ordre établi. Raymond Barre, Premier ministre français à cette époque, ne voyait pas moins que le germe puissant de l'anarchie et de la subversion dans ce mouvement.

Dans ce contexte aussi, la région, le local, la petite dimension de manière plus générale, sont l'objet de nouvelles attentions, investis de valeurs intrinsèquement positives. "De grand, de révolutionnaire, il n'y a que le mineur » proclamaient les militants radiophoniques de Radio Alice ${ }^{2}$. C'est le temps des " révolutions minuscules ${ }^{3}$ et le temps du reflux ou de la réfutation des idéologies traditionnelles. Les micro-sujets se substituaient aux macro-théories. Ces courants rejoignaient par là les revendications régionales ou régionalistes qui étaient un autre des traits notables de l'époque.

Dans l'esprit des promoteurs des radios libres, ce que les mass media ne pouvaient ou ne voulaient pas faire, les micro-médias devraient et allaient le réaliser. On en finirait enfin avec la notion d'un public de masse, anonyme et amorphe, arrosé par des parapluies médiatiques démesurés. Le local, les régions, les identités et cultures attenantes, les notions de pays, de terroirs, l'emploi des langues régionales étaient des éléments qui revenaient souvent et formaient une partie importante du socle idéologique sur lequel s'appuyaient les militants des premières radios libres. En fait, les radios libres étaient des radios locales, en bonne partie parce que la technologie qu'elles employaient

1 Dominique Wolton, Éloge du grand public, une théorie critique de la télévision, Paris, Champs Flammarion, 1993, p. 80-96.

2 Collectif A/TRAVERSO, Radio Alice, radio libre, Paris : Jean Pierre Delarge éditeur, 1977, p. 67.

3 «Les Révolutions minuscules », Autrement, $n^{\circ}$ 29, Février 1981 
les y contraignait. L'usage de la modulation de fréquence leur imposait une zone de diffusion réduite. Mais, en se saisissant du thème du local, ces radios en France, comme en Italie, se forgeaient un argument de poids contre le monopole ; elles pouvaient se définir comme complémentaires des stations nationales existantes et non comme concurrentes immédiates ${ }^{1}$.

D'un point de vue social, l'espoir placé dans les radios libres était qu'elles permettent à des minorités, à des groupes, à des communautés spécifiques, par qui et pour qui elles auraient été conçues, d'avoir accès à des médias qui leur soient propres, qui leur permettent de cultiver leurs identités tout en les faisant connaître aux autres. Il s'y ajoutait une dimension libératoire induite. Il s'agissait de rendre la parole aux exclus, à ceux qui jusque-là se taisaient. Les militants des radios libres prônaient une conquête des mécanismes de la radiodiffusion pour en finir avec la division et la hiérarchie des tâches, l'appropriation du micro et de la parole pour rompre avec une idéologie et des privilèges professionnels. Il fallait "faire sauter le filtre du journalisme professionnel qui stérilise le vécu, faire sauter le filtre du langage politique qui se pose en savoir, faire sauter les canons d'une esthétique apprise pour la détourner dans ces pratiques multiples qui créeront leur propre langage ${ }^{2}$. Cet objectif, on le retrouvait par exemple dans le projet de Radio Périgueux 103, en France, en 1981. La station se devait d'exister disait-elle pour donner "la parole aux gens de la rue, en dehors de la médiation des techniciens, spécialistes, intellectuels » qui eux disposaient déjà exclusivement de ce privilège. L'usage direct de la radio participait d'une démarche de démystification des médias quant à leur objectivité, leur neutralité. Il devait contribuer à ruiner leur pouvoir d'imposition symbolique ${ }^{3}$.

La restitution aux récepteurs de leurs rôles et capacités de réponse est l'un des premiers axes autour desquels s'articulent les discours sur la communication alternative. Convertir le récepteur en émetteur est le premier défi auquel veulent se confronter ces expériences. Tout le monde doit pouvoir venir parler et intervenir, à tout moment en présence

1 Patrice Flichy, « La explosión del monólogo, las radios paralelas en la Europa occidental », p. 180-188, in De las ondas rojas a las radios libres, textos para la historia de la radio / Lluís Bassets ed., Barcelona, Gustavo Gili, 1981

2 Collectif Radios Libres Populaires, Les Radios libres, Paris : Maspero 1978, p. 24.

3 Jean-Jacques Cheval, Les Radios en France ; Histoire, état et enjeux, Rennes, Apogée, 1997. 
ou par téléphone. Dans la communauté, le quartier, le village, l'entreprise, les auditeurs doivent devenir les principales sources d'information des stations, l'information étant elle-même toujours prioritaire. C'est l'ensemble des programmes des radios libres qui doit participer, se mêler aux mouvements et luttes des associations et groupes sociaux marginalisés.

Dans le même temps, les changements visent l'instrument luimême et son contenu qui ne sont plus distingués. "Si le sujet qui parle se transforme, alors la forme, l'instrument, le mode de production, circulation, réception du message doit changer ${ }^{1}$. Les radios libres ne doivent pas, par exemple, avoir de grille de programme fixe. L'improvisation, la fête, le « happening » permanent sont des horizons rêvés. La technologie elle-même est contestée. En 1978, on considérait trop élevée la somme de 5000 francs [760 Euros)] à investir dans un émetteur. Plusieurs ouvrages et publications proposaient des alternatives artisanales et s'accompagnaient de chapitres techniques où l'on pouvait apprendre à construire son propre émetteur.

Le fonctionnement et l'organisation des nouvelles structures médiatiques devaient participer eux aussi à l'altérité des projets. Le préalable était que les formes de communication alternative soient soustraites à l'économie de marché. En conséquence, la radiodiffusion privée, qualifiée de commerciale, était rejetée, stigmatisée en bloc. La propriété collective des médias devait être le gage de leur fonctionnement démocratique.

La radio libre non lucrative, nouvelle agora, nouveau forum - véritables mots-clés de cette époque - appartenant à tous, investie par tous, devient le centre de communication de la communauté pour laquelle elle existe, de la population à laquelle elle s'adresse. Avant l'évocation de la démocratie électronique, les militants des radios libres d'intervention rêvent d'édifier une place publique politique, terrain d'une démocratie hertzienne revisitée. Ils prônaient une conception politiquement engagée d'une radio totalement alternative et novatrice, avec une générosité et un volontarisme certains, mais aussi la naïveté et les illusions lyriques dont on a pu souvent juger au prisme de la réalité.

Dans nombre de pays européens, là où elles furent finalement autorisées, un grand nombre de radios libres sont simplement devenues des radios privées. Les exemples italiens en sont de beaux exemples.

1 Collectif A/Traverso, Op.cit., 1977, p. 87. 
À peine nées, tolérées ou autorisées, elles se sont vite transformées et parfois bien loin des rêves et attentes que suscitaient les radios libres, souvent au profit d'une normalisation aussi bien culturelle, symbolique qu'économique. Prendre la mesure de cette évolution objective est une chose, mais ne soustrait pas à s'interroger sur ses causes. Une première erreur d'évidence serait d'opposer l'utopie à la réalité, le passé au présent et l'époque rêvée des radios libres, conviviales, populaires et de proximité aux radios privées, aux réseaux commerciaux et aux chaînes d'aujourd'hui. Il faut avoir à l'esprit que la situation présente des radios n'est pas plus uniforme que celle d'hier. Au-delà des discours militants dominants, les projets commerciaux étaient déjà là et parmi les premières radios libres. Dès l'origine, les décalages entre les discours et la réalité étaient patents.

En tout état de cause, les radios militantes, les stations qui voulaient bouleverser les systèmes locaux d'information, ont rencontré comme première difficulté celle de rassembler spontanément un public nombreux et légitimant. Il est apparu qu'il ne suffisait pas de se proclamer radios populaires pour toucher mécaniquement et obligatoirement un auditoire du même nom. Les vertus démocratiques intrinsèques de l'expression spontanée de tous et de chacun à tout moment se révélaient à l'usage moins évidentes qu'elles ne semblaient l'être quand elles étaient seulement projetées ou interdites. La prise de parole libératrice apparaissait difficile à mettre en œuvre.

"On a eu tout à coup la possibilité de tout dire à tout le monde; mais à la réflexion, il n'avait rien à lui dire ». Dans sa cruelle sévérité, cette autre sentence de Bertolt Brecht, appliquée aux premières années de la radiodiffusion, semblait assez s'accorder à nombre de projets des années 80 et ensuite. Ce fut vrai en France et ailleurs.

Par ailleurs, concentrés sur des aspects techniques et les limites politiques ou judiciares, les militants des médias alternatifs oubliaient les causes sociales des inégalités, face à l'information et à la communication. Sans généraliser systématiquement, on peut aisément reprendre ce qu'écrivait Paul Beaud, à propos des télévisions communautaires québécoises, pour le compte d'autres expériences de médias alternatifs. "Si le mythe d'une autre télévision, faite par et pour son public, n'a guère survécu à son expérimentation, les raisons doivent en être aussi recherchées ailleurs que dans l'économie ou la micro-politique locale: dans leur échec à modifier les structures de la communication sociale et donc aussi du pouvoir. Échec au demeurant prévisible. Comme tout autre proclamation abstraite de ce genre, le "droit à la communica- 
tion » des idéologues des media communautaires (...) fait table rase non des inégalités que ce droit entend abolir mais de leurs origines sociales ${ }^{1}$. Selon cet auteur, les militants d'une radiodiffusion alternative méconnaissaient que "les médias ne sont pas que l'enjeu d'un processus de la lutte des classes, [mais qu'] ils en sont aussi le lieu » 2. En Europe, comme en Amérique du Nord, les notions de médias alternatifs s'adressant à des communautés, à des petites localités, à des groupes peu différenciés socialement ou sans contradiction apparente, induisaient de telles conceptions. La revalorisation du local, tout comme l'idéologie du développement individuel, devenait l'inspiration et la source d'un nouveau, et improbable, consensus social ${ }^{3}$.

L'usage social des radios libres est tout autant à rechercher à l'intérieur même des stations que dans leur réception auprès des publics. L'accès libre potentiel à l'antenne ne devrait pas faire perdre de vue que l'ensemble de la population n'avait nullement un accès illimité au micro. "Le problème est que "prendre la parole », c'est souvent la prendre à la place de quelqu'un, quand bien même on dit vouloir la lui redonner", notait Paul Beaud, or «la rhétorique n'est [pas] l'arme des défavorisés. Silencieuse, la majorité va le rester ${ }^{4}$. La pertinence des analyses du sociologue Pierre Bourdieu sur «ce que parler veut dire » ${ }^{5}$, ne pouvait qu'être confirmée dans des structures où précisément régnait le verbe. À la radio comme ailleurs et peut-être plus encore qu'ailleurs parce que la parole est le signe majeur de distinction, l'expression orale révèle les inégalités de ceux qui s'y prêtent. Pierre Bourdieu écrit « [...] fonder sur le chiffrement et le déchiffrement, donc sur la mise en ouvre d'un code, ou d'une compétence génératrice, l'échange linguistique est aussi un échange économique, qui s'établit dans un certain rapport de forces symbolique entre un producteur, pourvu d'un certain capital linguistique, et un consommateur (ou un marché) [...]. Autrement dit, les discours ne sont pas seulement (ou seulement par exception) des

1 Paul Beaud, La société de connivence, média, médiations et classes sociales, Paris, Aubier, 1984, p. 260.

2 Jean-Marie Piemme, La télévision comme on la parle, Bruxelles, Editions Labor/ Nathan, 1978, p. 26.

3 Michel Sénécal, Une Démocratie du paradoxe, logique d'acteurs et démocratisation de l'espace médiatique, Rennes, Université de Rennes 2, Thèse d'Université en Sciences de l'Information et de la Communication, 1992, p. 335.

4 Paul Beaud, Op. cit., p. 276.

5 Pierre Bourdieu, Ce que parler veut dire. L'économie des échanges linguistiques, Paris, Fayard, 1982. 
signes destinés à être compris, déchiffrés : ce sont des signes de richesse destinés à être évalués, appréciés et des signes d'autorité, destinés à être crus et obéis ${ }^{1}$.

On a pu le constater en France et ailleurs, les médias « communautaires », les radios « libres » furent bien l'enjeu d'une appropriation sociale collective et qui ne fut pas qu'individuelle. L'une des raisons majeures de la création de milliers de radios locales privées est sans doute à rechercher dans une forte aspiration à entrer dans le monde des médias et de la communication. Aspiration individuelle de passionnés de la radio qui des années durant se sont dépensés sans compter pour réaliser ce rêve. Mais, il s'agissait parallèlement d'une aspiration collective qui pouvait dépasser la conscience individuelle de ses acteurs. Ce n'est pas non plus une coïncidence si elles sont nées et se sont affirmées autour de mai 1981, quand, concrétisés par l'alternance politique, l'accès et la participation au pouvoir paraissaient s'ouvrir à ces nouvelles couches sociales. On peut avancer l'hypothèse que le mouvement des radios libres, de celles qu'on a appelé ensuite les radios locales privées a été surinvestie par une catégorie sociale que l'on pouvait baptiser "nouvelle petite bourgeoisie », "classe intermédiaire », "classe moyenne » ou autre selon des terminologies ayant précisément évoluées dans cette période. Quoiqu'il en soit, il s'agissait d'un groupe social en mal de repères, de définition, de reconnaissance, d'ascension ou de pérennisation sociale. Il regroupait des individus qui souhaitaient trouver dans les associations, les comités d'usagers et de consommateurs, l' animation socioculturelle et in fine la communication et les médias, le témoignage "de son entrée collective dans l'espace public, dans l'historicité de la société » et par là même les moyens de leur propre production sociale, l'espoir d'une reproduction en tant que classe $^{2}$. Les radios libres françaises ont présenté la particularité exemplaire de se situer au carrefour de tous ces lieux.

D'un autre point de vue, il ne faut pas écarter les stratégies purement politiques. En France encore, mais ceci peut valoir ailleurs, les choix de 1981, qui consistaient à modifier le système de communication français par le bas n'était pas anodin. Il y avait certainement là un moyen de satisfaire la base sociale du nouveau pouvoir dont l'élection reposait en grande partie sur le ralliement des couches moyennes et intellectuelles évoquées et investies dans le mouvement des radios

1 Pierre Bourdieu, Langage et Pouvoir symbolique, Paris, Points Seuil, 2001, p. 99.

2 Paul Beaud, op. cit., p. 248 et p. 298-299. 
libres. L'ouverture d'un secteur pluraliste d'information et de communication à la base, à l'échelle exclusivement locale, dispensait le nouveau pouvoir de repenser immédiatement le système d'information plus largement ou plus globalement et particulièrement à son sommet. Mais, la radiodiffusion ne formait pas un champ clos, très tôt la dérégulation du système audiovisuel français entamée par la radiodiffusion a été étendue à la télévision, porteuse d'enjeux jugés beaucoup plus cruciaux et très vite les radios libres, la radiodiffusion même dans son ensemble sont passées, à nouveau, au second plan. Les télévisions locales privées, puis les télévisions privées nationales, ou leur privatisation, ont relégué les radios associatives au rang de domaine secondaire, au moment où ce qui était en jeu était l'appropriation des industries de l'information et quand triomphait l'idéologie confuse, messianique et quasi « thaumaturgique » de la communication des années 1980.

Il reste que du mouvement des radios libres sont nés, ou naissent encore, des paysages radiophoniques pluralistes. La diversité de nombreux systèmes radiophoniques européens est certainement redevable aux militants des radios alternatives qui ont pu faire éclater des systèmes audiovisuels monopolistiques et sclérosés.

Ce pluralisme est-il au moins synonyme d'accès démocratique aux programmes, à des contenus riches et diversifiés et à des dimensions culturelles élargies pour tous. Il s'agit là d'autres aspects bien réels des enjeux démocratiques des dimensions et expressions médiatiques. La multiplicité des programmes radiophoniques, leur fragmentation, la dispersion des opérateurs radiophoniques que l'on constate à travers de nombreux pays européens signifient-ils un pluralisme de l'accès à la culture.

Si l'on s'en tient à la dimension musicale, dimension essentielle pour la radio, on peut en douter. Des voix nombreuses s'accordent pour dire la banalité, le conformisme, l'uniformité des productions radiophoniques trop souvent réduites à la duplication de quelques formats imités d'une région à l'autre, d'un pays à l'autre ; multiplicité ne rimant pas toujours avec diversité. Pierre Henry, compositeur et homme de radio, l'inventeur de la musique concrète rendait hommage à la radio : "Sans la radio, ma musique n'aurait jamais pu exister [...] elle était le moteur de trouvailles. À l'époque, c'était comme du cinéma sans l'image ». Mais il avouait qu'aujourd'hui il éteignait souvent son poste de radio ; désabusé par l'offre actuelle de programme : "Je suis de plus 
en plus réfractaire aux sons actuels. On ne les entend plus parce qu'on les entend trop $» 1$.

Pourtant, au sujet de la radio et des radios libres en particulier, il faut bien sûr se méfier de la condamnation implicite que renferme tout constat d'échec totalisant qui devient facilement le constat d'une impossibilité rédhibitoire au changement et aux transformations dans l'information et la communication et, par extension, le constat d'échec de quelque progrès que ce soit allant dans le sens d'une démocratisation accrue des moyens d'information. Or ces stations demeurent, elles ont su inscrire leur action dans la durée, rassembler des énergies, s'implanter dans le paysage local, obtenir une légitimité, par leurs pratiques quotidiennes et tout simplement leur présence sur le terrain.

Le troisième secteur radiophonique, associatif, non étatique et non commercial existe en France, en Europe et à travers le monde. Il a même tendance à s'étendre. La potentialité d'expression et d'actions, que l'on peut qualifier de démocratique, qu'il représente n'est pas que virtuel. Si l'on continue de penser que "l'idée d'un projet social du partage démocratique de l'espace médiatique ${ }^{2}$ reste valide ou, qu'inversement, la définition des utilisations des médias figure bien parmi les enjeux de la démocratie, alors la défense de l'intervalle encore occupé par les radios locales héritières des radios libres reste valide.

Leur existence même milite toujours pour une possible alternative aux moyens de communication de masse. Par leur présence, elles témoignent d'une réelle résistance aux modèles dominants et constituent une ouverture, étroite sans doute, et conflictuelle ou encore inégalitaire, mais qui figure le droit d'entrée des citoyens au monde médiatique. Au Québec, au Canada, certains auteurs considéraient que «la radiodiffusion communautaire est une composante à part entière $d u$ système [médiatique] et qu'elle devrait à ce titre jouer le rôle de porte d'entrée ou de garante du droit d'accès au système " ${ }^{3}$. La radio dans ses variantes associatives, communautaires, libres, entre autres, peut être à même de jouer ce rôle.

Les stations de cette nature constituent un apport essentiel à la diversité médiatique quand bien même la place qui leur est réservée au

1 Pierre Henry, Télérama, 19 mars 2005, n² 2879, p. 163

2 Michel Sénécal, Op. Cit., 1992, p. 275-364.

3 G. Caplan, F. Sauvageau, Rapport du groupe de travail sur la politique de la radiodiffusion, Ministère des Approvisionnements et services Canada, 1986, p. 549. 
sein des systèmes radiophoniques nationaux varie, selon les pays, allant de la présence marginale à la reconnaissance significative.

En 2007, la section Amérique latine de l'association mondiale AMARC (Association Mondiale des Radiodiffuseurs Communautaires), à la suite d'une consultation internationale, initiée à Montevideo en Uruguay, et une réflexion de plusieurs mois, vient de réaffirmer solennellement ce que doivent être les « Principes pour une législation démocratique sur la radiodiffusion communautaire », qu'elle entend propager et, si possible imposer, à travers les différents pays du continent sud américain. Il est notamment précisé que «La diversité et le pluralisme au sein de la radiodiffusion est le principal objectif de tout cadre régulateur d'une radio démocratique. Ceci implique l'existence de mesures effectives pour empêcher la concentration dans la propriété des médias, garantir l'expression d'une diversité des contenus et des opinons au sein des médias, et reconnaître une diversité de formes juridiques pour la propriété, la finalité et la logique de fonctionnement des médias, avec trois secteurs ou modalités de radiodiffusion différenciées : publique étatique, commerciale - privée et sociale sans but lucratif où s'inscrivent les médias proprement communautaires ». Au delà de la radio, cette organisation affiche ses objectifs, il s'agit de démocratiser la parole pour démocratiser la société ${ }^{1}$. Qu'on les nomme radios communautaires ou radios associatives, ces stations sont des lieux d'expression, d'apprentissage, de participation. Par l'accès qu'elles permettent à une expression médiatisée, un usage des technologies, elles favorisent un apprentissage aux médias. À elles seules, elles ne bouleversent sans doute pas les inégalités face au langage et à l'expression, mais au moins elles contribuent à désacraliser le pouvoir symbolique d'imposition des moyens et modes d'information dominants et participent à une approche banalisée de ceux-ci. En cela, elles réalisent, au moins partiellement, les objectifs initiaux de démocratisation énoncés et deviennent ou demeurent une utopie pratique démocratique.

1 « Democratizar la palabra para democratizar la sociedad», http://alc.amarc.org/ 
